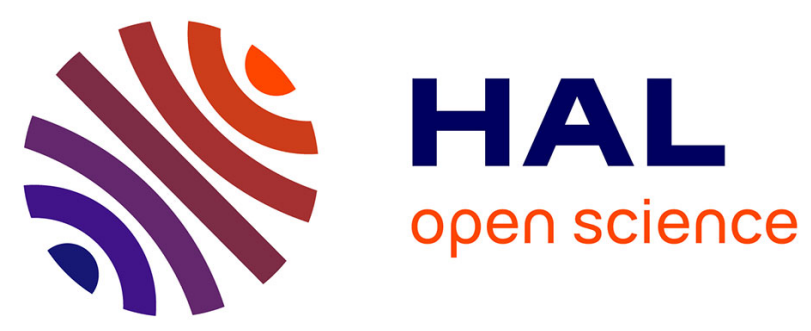

\title{
Teleoperation of a Surgical Robot via Airborne Wireless Radio and Transatlantic Internet Links
}

\author{
Mitchell J. Lum H., Diana C. Friedman W., Hawkeye H. King I., Regina \\ Donlin, Ganesh Sankaranarayanan, Timothy Broderick J., Mika Sinanan N., \\ Jacob Rosen, Blake Hannaford
}

\section{To cite this version:}

Mitchell J. Lum H., Diana C. Friedman W., Hawkeye H. King I., Regina Donlin, Ganesh Sankaranarayanan, et al.. Teleoperation of a Surgical Robot via Airborne Wireless Radio and Transatlantic Internet Links. 6th International Conference on Field and Service Robotics - FSR 2007, Jul 2007, Chamonix, France. inria-00199663

\section{HAL Id: inria-00199663 https://hal.inria.fr/inria-00199663}

Submitted on 19 Dec 2007

HAL is a multi-disciplinary open access archive for the deposit and dissemination of scientific research documents, whether they are published or not. The documents may come from teaching and research institutions in France or abroad, or from public or private research centers.
L'archive ouverte pluridisciplinaire HAL, est destinée au dépôt et à la diffusion de documents scientifiques de niveau recherche, publiés ou non, émanant des établissements d'enseignement et de recherche français ou étrangers, des laboratoires publics ou privés. 


\title{
Teleoperation of a Surgical Robot via Airborne Wireless Radio and Transatlantic Internet Links
}

\author{
M.J.H. Lum ${ }^{1}$, D.C.W. Friedman ${ }^{1}$, H.H.I. King ${ }^{1}$, R. Donlin ${ }^{1}$, G. \\ Sankaranarayanan $^{1}$, T.J. Broderick ${ }^{2}$, M.N. Sinanan ${ }^{1}$, J. Rosen ${ }^{1}$, and B. \\ Hannaford $^{1}$ \\ 1 University of Washington, BioRobotics Lab, Seattle, WA, USA \{mitchlum, \\ dwarden, hawkeye1, rdonlin, ganeshs, mssurg, rosen, \\ blake\}@u. washington.edu \\ 2 University of Cincinnati, Center for Surgical Innovation, Cincinnati, OH, USA \\ brodertj@ucmail.uc.edu
}

Summary. Robotic assisted surgery generates the possibility of remote operation between surgeon and patient. We need better understanding of the engineering issues involved in operating a surgical robot in remote locations and through novel communication links between surgeon and surgery site. This paper describes two recent experiments in which we tested the RAVEN, a new prototype surgical robot manipulation system, in field and laboratory conditions. In the first experiment, the RAVEN was deployed in a pasture and ran on generator power. Telecommunication with the surgical control station was provided by a novel airborne radio link supported by an unmanned aerial vehicle. In the second experiment, the RAVEN was teleoperated via Internet between Imperial College in London and the BioRobotics Lab at the University of Washington in Seattle. Data are reported on surgeon completion times for basic tasks and on network latency experience. The results are a small step towards teleoperated surgical robots which can be rapidly deployed in emergency situations in the field.

\section{Introduction}

Remote environments limit access to power and telecommunication resources needed by telesurgery systems. Mobile Robotic Telesurgery (MRT) systems that allow a remote surgeon to operate on a patient, regardless of their location or environment, need to overcome these resource limitations. In June 2006, we conducted experiments in pasture land outside Simi Valley, California using a novel MRT system comprised of the RAVEN surgical robot and an Unmanned Aerial Vehicle (UAV)-based communication system. The primary objective of this project was to demonstrate in the field a MRT system that would 
eventually allow a remote surgeon to operate on a patient regardless of their location or environment.

Network limitations including latency, bandwidth, jitter, packet loss and loss of signal, make telesurgical intervention in extreme environments difficult. While other existing topologies such as geosynchronous satellites can provide wireless communication, long latency precludes robust use of satellite communications in telesurgery. Geosynchronus orbit is $35,900 \mathrm{~km}$ above the earth's surface, and a complete loop from surgeon to robot and back requires two trips up and down: a 0.48 second round trip at the speed of light.

Various types of UAVs can operate at altitudes from 100 to 20,000 meters and carry equipment to provide robust, low-latency, high quality communications. In these experiments, the communication link was provided by a wireless digital datalink onboard a Puma UAV by AeroVironment Inc. (Simi Valley, CA). The Puma is a 2-meter wingspan, Small Unmanned Aerial Vehicle (SUAV) that flies at altitudes below 5000 meters above sea level and can provide line-of-sight communication up to a distance of $12 \mathrm{~km}$ with low gain antennas and $20 \mathrm{~km}$ with higher gain antennas. Approximately 4,000 Pumas are currently deployed across the world in military applications.

Literature Review The earliest teleoperators, invented by Ray Goertz of Argonne National Labs in the late 1940's and early 1950's [1] are mechanical hands, coupled through a system of cables and pulleys to a remote handle which the operator can control at a safe distance. These mechanisms were very effective and are still in use, but are limited by their mechanical nature to short distances and similar size scales.

After continuous development at many labs [2, 3], teleoperation technology has matured enough in the last 15 years to be applied to surgery. By connecting the surgeon to the tools through teleoperators, the tools can be made smaller than the human hand, more dexterous in small body cavities, and, perhaps most revolutionary, can connect surgeon and patient across large distances.

In the early 1990's SRI International developed a two-handed teleoperated surgery unit for DARPA [4]. This highly influential project encouraged the startup of two companies to address the civilian surgery market, Computer Motion Inc. (Goleta, CA), and Intuitive Surgical Inc. (ISI) of Silicon Valley, CA. Both of these companies developed FDA approved surgical robot systems, the Zeus from Computer Motion and the da Vinci from ISI [5]. In 2003 they merged under the name of ISI. Over 300 da Vinci systems are in use around the world today. Both of these systems are teleoperators, but neither had the capability to separate the surgeon and patient by more than a few feet.

In 2001, a team led by Dr. Jacques Marescaux worked with Computer Motion to develop a specially modified Zeus system to perform the first remote surgical procedure (laparoscopic cholecystectomy) on a human patient [6, 7]. Dr. Marescaux controlled the robot from New York, and the patient was in Strasbourg, France. Using a similarly modified Zeus, Dr. Mehran Anvari of McMaster University, has treated about 25 patients in Northern Canada 
from his offices in southern Ontario [8]. Recently, Dr. Timothy Broderick has evaluated remote surgical technology in extreme environments. With support from the US Army and NASA, in 2005 he completed the NEEMO 9 mission - a two week stay in the Aquarius undersea habitat, maintained by the US National Oceanographic and Atmospheric Administration (NOAA), 19 meters underwater off the Florida Keys. An updated version of SRI's manipulator was deployed in this cramped habitat and Dr. Anvari successfully operated the robot from Canada with up to 2 seconds of time delay [9], performing simulated surgical procedures and the handling of simulated lunar rocks.

A major user of remote surgical technologies could be the military. Can combat casualties be reduced or ameliorated by getting surgical care to the soldier faster than getting the soldier to the care? Surgeons are a scarce resource in the military; with remote surgical technology they could be rapidly deployed right to where they are needed, switching electronically from one battlefield to another in seconds, without the need to "scrub in".

The world has seen a tremendous loss of life due to natural disasters. The scope of MRT applications will reach the civillian market as well, providing emergency medical care to disaster zones. Rather than a limited number of surgeons and emergency medical personel working non-stop in the disaster area, remote surgery relief stations could be deployed to the region and surgeons from around the world could connect in, providing trauma and other critical healthcare.

The need for small and deployable surgical telerobotic systems led to recent development of a new surgical robot, the RAVEN, by the authors at the University of Washington [10]. This manuscript presents experiences from the first field trials. The extreme environment provided difficulties that resulted in little time for rigorous evaluation of the quality of surgical performance but did lay the initial ground work for future experiments.

\section{Methods}

RAVEN Surgical Robot System A detailed discussion of the RAVEN's design was reported in Feruary 2006 [10]. The system consists of a patient site (slave), a surgeon site (master) and a communication link between them. The patient site (Figure 1) consists of two surgical manipulators that are positioned over the patient by passive macro-positioning arms. The surgeon site consists of a surgeon console featuring two PHANToM Omni devices to control each of the surgical manipulators, a foot pedal, a surgeon's interface screen and a video feed from the patient site. The communication link between patient and surgeon sites is a standard Internet connection using UDP connectionless protocol for minimum time delay.

Field readiness preparations Many research systems are conceived, developed and tested in a lab environment. To fully demonstrate the applicability of a system it must be deployed into the environment for which it 


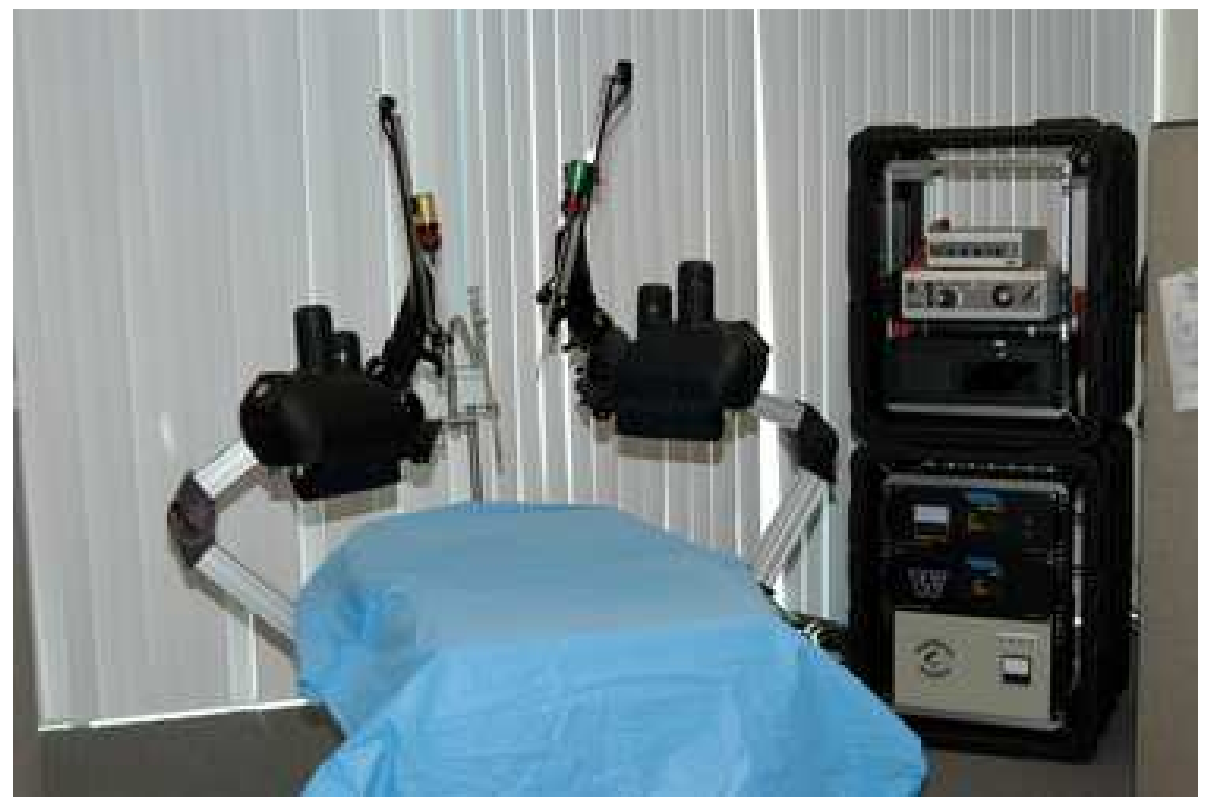

Fig. 1. Photograph of the surgical robot system being checked and tested at AeroVironment's headquarters prior to field deployment.

was designed. The ability to provide surgical care in rural, remote or extreme environments will allow surgeons to provide immediate care in battlefield or disaster emergencies. Thus, the RAVEN must not only operate in field conditions but must also be easy to transport and quick to set up.

In the lab, the RAVEN is set up on a fully articulated operating table that is large, heavy, and cumbersome. For field testing the RAVEN manipulators are mounted on a small portable operating table, fabricated from modular aluminum extrusions. It was rigid and heavy enough to be very stable, but light enough that two people could easily move it. The table has adjustable mounting locations for the positioning arms, creating a quickly adjustable platform upon which the surgical manipulators and the "patient" (Figure 2) were mounted.

Power-off brakes on the surgical manipulators are a key piece of the safety system. If the system loses power the brakes will automatically engage, keeping the manipulators' tools from falling due to gravity. However, during packing/unpacking and setup it is necessary to reorient the arms of the surgical manipulators. A battery override provides a simple and inexpensive solution. On each manipulator a $1 / 8$ " headphone jack was wired in parallel to existing brake circuitry. When the manipulators are disconnected, a $9 \mathrm{~V}$ battery connected to an $1 / 8$ " headphone plug can be used to power the brake cir- 
cuitry. This is particularly useful when removing the manipulators from the positioning arms and placing them in their transport cases.

The major evironmental concerns we expected to face in the desert included dust, heat and a lack of clean power. To protect the motor packs against dust and debris, 3-piece covers were rapid prototyped out of ABS plastic. Vents allow motor heat to dissipate and fan mounts were included so that active cooling from standard 2.5" computer fans could be added. In the field, the whole system was powered by construction grade internal combustion $110 \mathrm{~V}$ electric generators. To protect the computers and sensitive electronics from generator power spikes, a $1200 \mathrm{~W}$ line regulator from APC was used at the patient site.

For safe transport of the system, custom foam lined cases for the surgical manipulators, surgical tools, and the master console devices were obtained from H.H. Bauer Cases (Vancouver, WA). The major electronic components, including computers, power supplies, Maxon brushless motor amplifier and our USB2.0 interface device, were mounted inside two SKB Industrial RotoShock Rack cases. The Roto-Shock cases provide shock isolation between the components, mounted to its internal frame, and the exterior hard-shell. The entire system, including the two surgical manpulators, portable OR table, SKB cases, surgeon console, tools and back-up equipment, fit easily in a Chevy Express Cargo van. The system could be deployed and set up in under an hour.

Experimental Protocol An experimental protocol was developed to demonstrate system dexterity including touching a series of landmarks on a latex glove stretched over a box (Figure 2). The gloved box was marked with a circle, and a grid of 10 landmarks spaced $1 \mathrm{~cm}$ apart left to right and $0.5 \mathrm{~cm}$ apart toward and away from the camera. The landmarks were numbered 1-10 row by row starting with 1 at the upper left, and finishing with 10 at the lower right. The following five tasks were part of the experimental protocol.

1. Right hand touch each landmark in numeric order.

2. Left hand touch each landmark in numeric order.

3. Touch each landmark in numeric order using alternating left and right hands. Right hand touches the odd numbered landmarks, left hand touches the even numbered ones.

4. Right hand trace inner edge of circle clockwise.

5. Left hand trace inner edge of circle clockwise.

The tasks on the gloved box tested both the surgeon and manipulators ability to perform bi-manual gross positioning tasks. Beyond the experimental protocol, a surgeon performed intracorporeal knot tying (suturing) on the gloved box to demonstrate dexterous manipulation. 


\section{Experiments}

Telesurgery Field Experiment: Simi Valley, CA In early June of 2006, the RAVEN was driven from Seattle, WA and deployed and tested a few kilometers north of Simi Valley, CA for telesurgery experiments on an inanimate model.

The fielded system consisted of two stations connected by a wireless datalink relayed by UAV (Figure 3). The system was powered by generators and was set up under portable tents in an isolated field. Separated by a distance of 100 meters, the patient site (surgical manipulators) and the surgeon site (master control console) were connected via a wireless digital datalink relayed by AeroVironment's PUMA unmanned aircraft up to $1 \mathrm{~km}$ away at an altitude of about 100 meters. The datalink provided by AeroVironment utilized Internet Protocol communication at a rate of $1 \mathrm{MB}$ per second between the two sites allowing the master/slave communication architecture to remain unmodified for this experiment.

A single NTSC color video signal was provided using a digital video camera which acquired a closeup picture of the patient site. A second video channel was sometimes used to display the overall operating environment. HaiVision Inc. (Montreal, Canada) provided a hardware codec that transmitted the video signal at 800kbps in MPEG-2 format.

During three days of field deployment, kinematic data of the surgeons' commands and the surgical manipulator motions were collected along with network characterization data as two surgeons performed the experimental protocol described above. Control information from the master side was transmitted at 100 packets per second in the field trial and 1000 packets per second in a later Transatlantic trial (below).

Transatlantic Experiment: Seattle, WA, USA to London, England In July 2006, the system was tested in a long distance teleoperation experiment in collaboration with Imperial College (London, England). At the surgeon site two PHANToM 6-DOF Premium haptic devices were equipped
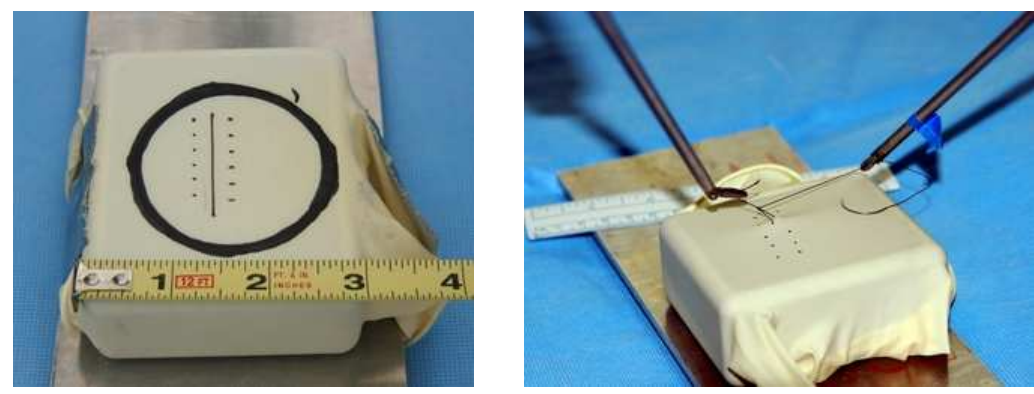

Fig. 2. (left) Experimental protocol was performed on a rubber glove stretched over a small box (right) Suture tied sucessfully on a latex glove. 


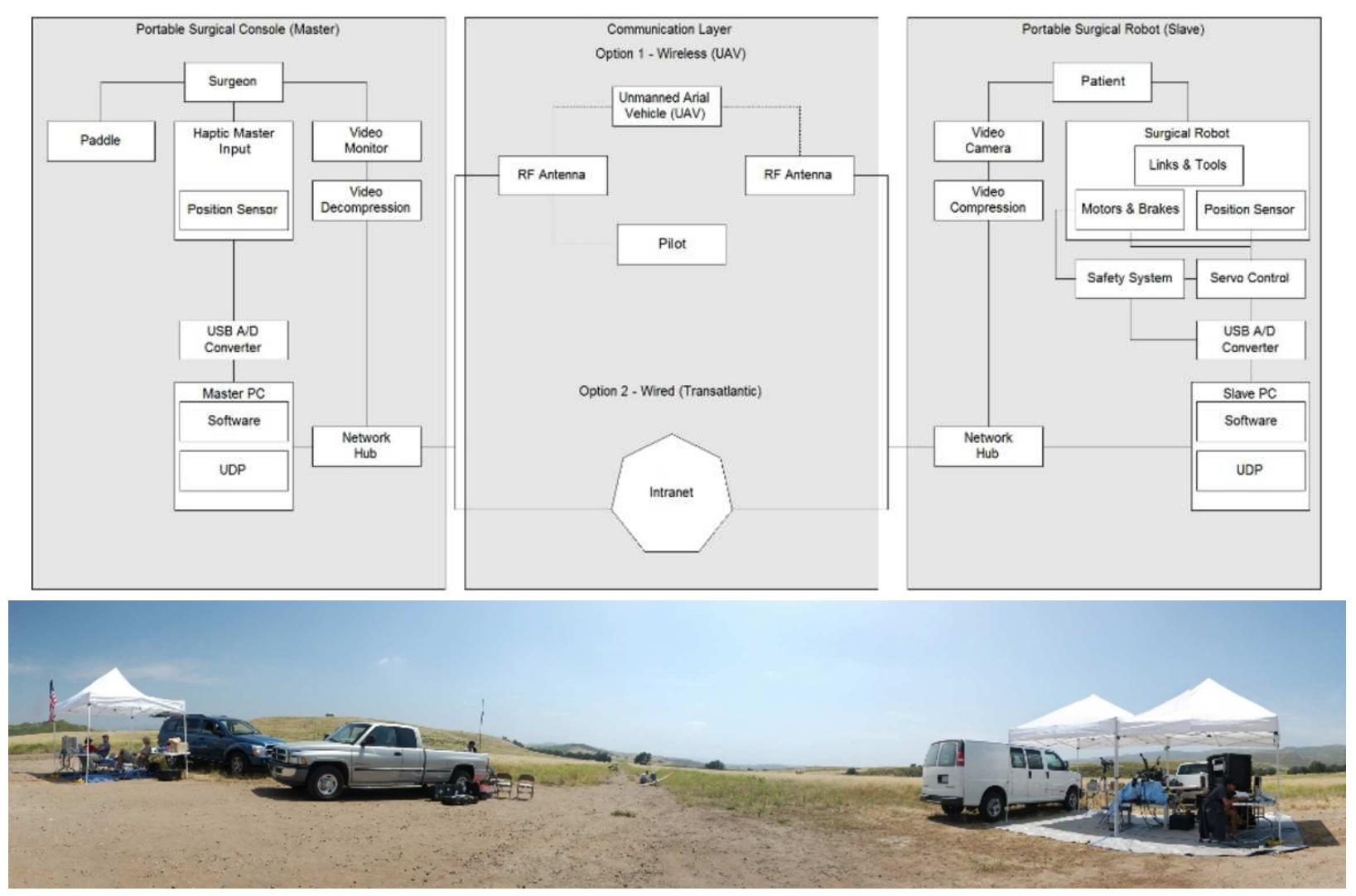

Fig. 3. Telesurgery experimental setup. (top) Block diagram of the system with UAV wireless link and wired Internet connection options. (bottom) Panoramic photo of the field site outside Simi Valley, CA. The surgeon site at left, patient site at right. UAV is visible in center of photo on ground. 
with our surgeon console software. iChat software (Apple Computer, Inc) was used for close-up video feedback of the surgical site. Skype video transmitted an overall view of the operating room. Audio, video, and RAVEN control signals were all sent through standard Internet. Two surgeons performed the same experimental protocol described above.

\section{Results}

The RAVEN system was originally designed so that the kinematic control signals would be sent from the surgeon site to the patient site at a $1 \mathrm{kHz}$ rate. During the field experiment packet loss through the UAV's wireless data link became problematic. When the kinematic control signals were transmitted at full rate the packet loss was approximately $80 \%$. The bandwidth of the system was scaled back so that kinematic control signals were sent at $100 \mathrm{~Hz}$ and video bandwidth was $800 \mathrm{kB} / \mathrm{s}$, resulting in reduced packet loss of between $3 \%-15 \%$. The surgeons did not notice any decrease in manipulator performance but did comment on increased pixelation in the video feed. While decreased video quality was noticable they did not feel it affected their task performance. During the transatlantic experiments no network bottlenecks were encoutered and the system was run at full bandwidth.

Table 1. Time delays in two telerobotic surgery configurations.

\begin{tabular}{lrr}
\hline Configuration & Network $(\mathrm{ms})$ & Video \\
\hline Wireless (UA)
\end{tabular}

Time delays for the two test configurations are given in Table 1. In the field experiment, with the UAV and HaiVision devices, the delays were substantially shorter. Internet latency (measured by ping) from Imperial College to our Lab in Seattle was about $140 \mathrm{~ms}$ and iChat video encoding/decoding delay was estimated at about 1 second. The transatlantic experiments showed both that the master console software was flexible enough to adapt to other PHANToM devices, and the RAVENs ability to teleoperate across long distances.

Task performance data for the four surgeons in two experiments are collected in Table 2. The variations between individual surgeons are pronounced compared to those between the two experimental conditions: field trials ("F") and Transatlantic Internet link ("T"). An example trajectory from one of the surgeons for Task 4 from the transatlantic Internet experiment is plotted in Figure 4. Further experiments must be performed to generate statistically significant results. 
Table 2. Task completion times (seconds) for two surgeons each in the two experiments ( $\mathrm{F}$ for field, and $\mathrm{T}$ for transatlantic).

\begin{tabular}{|c|c|c|}
\hline Subject & Exp. & $\begin{array}{lll}3 & 4 & 5\end{array}$ \\
\hline$\overline{1}$ & $\mathrm{~F}$ & $\begin{array}{lll}57 & 75 & 63\end{array}$ \\
\hline 2 & $\mathrm{~F}$ & 2131613236498 \\
\hline 3 & $\mathrm{~T}$ & $52118 \quad 11032 \quad 42$ \\
\hline 4 & $\mathrm{~T}$ & 173146430 \\
\hline
\end{tabular}

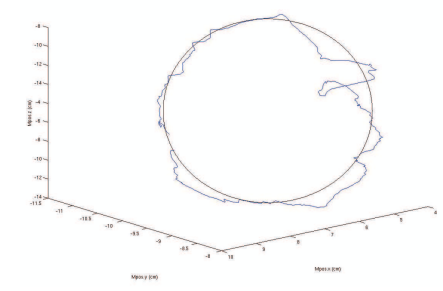

Fig. 4. Tool tip trajectory of surgeon performing Task 4 (see text) in the transatlantic Internet link experimment. Circle overlayed for perspective.

\section{Conclusions}

We have reported initial tests of a surgical robot in the field and when controlled over a global Internet link. Preliminary data indicate that under both conditions the robot can be effectively controlled in basic positioning tasks. The rigors of the field test exposed some weaknesses such as low generator voltage output (which automatically shut down our power conditioners) and poor control of tool orientation due to mechanical interferences inside the mechanism (which have now been fixed).

For some applications, the $1-10 \mathrm{~km}$ range of the low altitude UAV will limit applicability. Future MRT experiments using a High Altitude Long Endurance (HALE) UAV (Aerovironment Inc.), to provide ranges on the order of 10-50 $\mathrm{km}$, will allow further reaching emergency care to areas where conventional telecommunication connection is unavailable.

A novel feature of our current system is a low cost highly portable surgeon user interface built entirely from commercially available hardware [11]. In our field trial, we obtained very high video quality using dedicated codec hardware. In our intercontinental trial, we substituted freely available video chatting software with surprisingly good results. Although it is unlikely that basic technology like iChat video will statisfy clinical surgeons, it provides a very convenient platform for experimentation. Our low cost surgeon console facilitated the ease with which we were able to establish our intercontinental teleoperation collaboration. It will allow for cost effective collaboration with many more labs in the future. 
Futher development of the RAVEN will focus on increasing robustness of the system and a more rigorous scientific method for evaluating telerobotic surgical tasks. Deploying the system into a field environment and executing these experiments demonstrated the feasibility of Mobile Robotic Telesurgery (MRT) in remote environments and with long communication links. Development in this area will allow patients in rural, remote or extreme environments to receive lifesaving surgical interventions.

Acknowledgements The authors would like to acknowledge support from the US Army TATRC program grant number W81XWH-05-2-0080, our colleagues, Brett Harnett, Charles Doarn and Lynn Huffman at the University of Cincinnati, Julian Leung, George Mylonas, Sir Ara Darzi and GhuangZhong Yang at Imperial College in London, those at AeroVironment, and HaiVision Inc., and contributions from Clint Bland, Hiroki Mizumi and Levi Miller in the BioRobotics Lab.

\section{References}

1. R.C. Goertz and W.M. Thompson. Electronically controlled manipulator. $\mathrm{Nu}$ cleonics, Nov 1954.

2. A. K. Bejczy. Sensors, controls, and man-machine interface for advanced teleoperation. Science, 208:1327-1335, June 1980.

3. A.K. Bejczy and Z. Szakaly. Universal computer control systems (uccs) for space telerobots. Proceedings, IEEE ICRA, 4, Mar 1987.

4. J.W. Hill, P.S. Green, J.F. Jensen, Y. Gorfu, and A.S. Shah. Telepresence surgery demonstration system. Proceedings, IEEE ICRA, 3, 05/08/1994 05/13/1994.

5. G.S. Guthart and J.K. Salisbury. The intuitive telesurgery system: Overview and application. Proceedings IEEE ICRA, April, 2000.

6. J. Marescaux. Transatlantic robot-assisted telesurgery. Nature, 413, Sept 2001.

7. J. Marescaux, J. Leroy, F. Rubino, M. Smith, M. Vix, M. Simone, and D. Mutter. Transcontinental robot-assisted remote telesurgery: Feasibility and potential applications advances in surgical technique. Annals of Surgery, 235(4), April 2002.

8. A. Pirisi. Telerobotics brings surgical skills to remote communities. The Lancet, 361(9371).

9. M. Anvari, T. Broderick, H. Stein, T. Chapman, M. Ghodoussi, D. W. Birch, C. Mckinley, P. Trudeau, S. Dutta, and C.H. Goldsmith. The impact of latency on surgical precision and task completion during robotic-assisted remote telepresence surgery. Computer Aided Surgery, 10(2), March 2005.

10. M.J.H. Lum, D. Trimble, J. Rosen, K. Fodero, H. King, G. Sankaranarayanan, J. Dosher, R. Leushke, B Martin-Anderson, M.N. Sinanan, and B. Hannaford. Multidisciplinary approach for developing a new minimally invasive surgical robot system. In BioRob Conference, Pisa, Italy, February 2006.

11. G. Sankaranarayanan, H.I. King, S.Y. Ko, M.J.H. Lum, D.C.W. Friedman, J. Rosen, and B. Hannaford. Portable surgery master station for mobile robotic telesurgery. In ROBOCOMM, Athens, Greece, 2007. 